\title{
Publisher's Note: First cross-correlation analysis of interferometric and resonant-bar gravitational-wave data for stochastic backgrounds [Phys. Rev. D 76, 022001 (2007)]
}

B Abbott, R. Abbott, R. Adhikari, J. Agresti, P. Ajith, B. Allen, R. Amin, S. B. Anderson, W. G. Anderson, M. Arain, M. Araya, H. Armandula, M. Ashley, S. Aston, P. Aufmuth, C. Aulbert, S. Babak, S. Ballmer, H. Bantilan, B. C. Barish, C. Barker, D. Barker, B. Barr, P. Barriga, M. A. Barton, K. Bayer, K. Belczynski, J. Betzwieser, P. T. Beyersdorf,

B. Bhawal, I. A. Bilenko, G. Billingsley, R. Biswas, E. Black, K. Blackburn, L. Blackburn, D. Blair, B. Bland, J. Bogenstahl, L. Bogue, R. Bork, V. Boschi, S. Bose, P. R. Brady, V. B. Braginsky, J. E. Brau, M. Brinkmann, A. Brooks, D. A. Brown, A. Bullington, A. Bunkowski, A. Buonanno, M. Burgamy, O. Burmeister, D. Busby, R. L. Byer, L. Cadonati, G. Cagnoli, J. B. Camp, J. Cannizzo, K. Cannon, C. A. Cantley, J. Cao, L. Cardenas, M. M. Casey, G. Castaldi, C. Cepeda, E. Chalkey, P. Charlton, S. Chatterji, S. Chelkowski, Y. Chen, F. Chiadini, D. Chin, E. Chin, J. Chow, N. Christensen, J. Clark, P. Cochrane, T. Cokelaer, C. N. Colacino, R. Coldwell, R. Conte, D. Cook, T. Corbitt, D. Coward, D. Coyne,

J. D. E. Creighton, T. D. Creighton, R. P. Croce, D. R. M. Crooks, A. M. Cruise, A. Cumming, J. Dalrymple,

E. D’Ambrosio, K. Danzmann, G. Davies, D. DeBra, J. Degallaix, M. Degree, T. Demma, V. Dergachev, S. Desai,

R. DeSalvo, S. Dhurandhar, M. Diaz, J. Dickson, A. Di Credico, G. Diederichs, A. Dietz, E. E. Doomes, R. W. P. Drever, J.-C. Dumas, R. J. Dupuis, J. G. Dwyer, P. Ehrens, E. Espinoza, T. Etzel, M. Evans, T. Evans, S. Fairhurst, Y. Fan, D. Fazi, M. M. Fejer, L. S. Finn, V. Fiumara, N. Fotopoulos, A. Franzen, K. Y. Franzen, A. Freise, R. Frey, T. Fricke, P. Fritschel, V. V. Frolov, M. Fyffe, V. Galdi, J. Garofoli, I. Gholami, J. A. Giaime, S. Giampanis, K. D. Giardina, K. Goda, E. Goetz, L. Goggin, G. Gonzalez, S. Gossler, A. Grant, S. Gras, C. Gray, M. Gray, J. Greenhalgh, A. M. Gretarsson, R. Grosso, H. Grote, S. Grunewald, M. Guenther, R. Gustafson, B. Hage, W. O. Hamilton, D. Hammer, C. Hanna, J. Hanson, J. Harms, G. Harry, E. Harstad, T. Hayler, J. Heefner, I. S. Heng, A. Heptonstall, M. Heurs, M. Hewitson, S. Hild, E. Hirose, D. Hoak, D. Hosken, J. Hough, E. Howell, D. Hoyland, S. H. Huttner, D. Ingram, E. Innerhofer, M. Ito, Y. Itoh, A. Ivanov, D. Jackrel, B. Johnson, W. W. Johnson, D. I. Jones, G. Jones, R. Jones, L. Ju, P. Kalmus, V. Kalogera, D. Kasprzyk, E. Katsavounidis, K. Kawabe, S. Kawamura, F. Kawazoe, W. Kells, D. G. Keppel, F. Ya. Khalili, C. Kim, P. King, J. S. Kissel, S. Klimenko,

K. Kokeyama, V. Kondrashov, R. K. Kopparapu, D. Kozak, B. Krishnan, P. Kwee, P. K. Lam, M. Landry, B. Lantz, A. Lazzarini, B. Lee, M. Lei, J. Leiner, V. Leonhardt, I. Leonor, K. Libbrecht, P. Lindquist, N. A. Lockerbie, M. Longo, M. Lormand, M. Lubinski, H. Luck, B. Machenschalk, M. MacInnis, M. Mageswaran, K. Mailand, M. Malec, V. Mandic,

S. Marano, S. Marka, J. Markowitz, E. Maros, I. Martin, J. N. Marx, K. Mason, L. Matone, V. Matta, N. Mavalvala,

R. McCarthy, B. J. McCaulley, D. E. McClelland, S. C. McGuire, M. McHugh, K. McKenzie, J. W. C. McNabb,

S. McWilliams, T. Meier, A. Melissinos, G. Mendell, R. A. Mercer, S. Meshkov, E. Messaritaki, C. J. Messenger, D. Meyers, E. Mikhailov, P. Miller, S. Mitra, V. P. Mitrofanov, G. Mitselmakher, R. Mittleman, O. Miyakawa, S. Mohanty, V. Moody, G. Moreno, K. Mossavi, C. MowLowry, A. Moylan, D. Mudge, G. Mueller, S. Mukherjee, H. Muller-Ebhardt, J. Munch, P. Murray, E. Myers, J. Myers, T. Nash, G. Newton, A. Nishizawa, K. Numata, B. O’Reilly, R. O’Shaughnessy, D. J. Ottaway, H. Overmier, B. J. Owen, H.-J. Paik, Y. Pan, M. A. Papa, V. Parameshwaraiah, P. Patel, M. Pedraza, S. Penn, V. Pierro, I. M. Pinto, M. Pitkin, H. Pletsch, M. V. Plissi, F. Postiglione, R. Prix, V. Quetschke, F. Raab, D. Rabeling, H. Radkins, R. Rahkola, N. Rainer, M. Rakhmanov, M. Ramsuder, K. Rawlins, S. Ray-Majumder, V. Re, H. Rehbein, S. Reid, D. H. Reitze, L. Ribichini, R. Riesen, K. Riles, B. Rivera, N. A. Robertson, C. Robinson, E. L. Robinson, S. Roddy, A. Rodriguez, A. M. Rogan, J. Rollins, J. D. Romano, J. Romie, R. Route, S. Rowan, A. Rudiger, L. Ruet, P. Russell, K. Ryan, S. Sakata, M. Samidi, L. Sancho de la Jordana, V. Sandberg, V. Sannibale, S. Saraf, P. Sarin, B. S. Sathyaprakash,

S. Sato, P. R. Saulson, R. Savage, P. Savov, S. Schediwy, R. Schilling, R. Schnabel, R. Schofield, B. F. Schutz,

P. Schwinberg, S. M. Scott, A. C. Searle, B. Sears, F. Seifert, D. Sellers, A. S. Sengupta, P. Shawhan, D. H. Shoemaker, A. Sibley, J. A. Sidles, X. Siemens, D. Sigg, S. Sinha, A. M. Sintes, B. J. J. Slagmolen, J. Slutsky, J. R. Smith, M. R. Smith, K. Somiya, K. A. Strain, D. M. Strom, A. Stuver, T.Z. Summerscales, K.-X. Sun, M. Sung, P. J. Sutton, H. Takahashi, D. B. Tanner, M. Tarallo, R. Taylor, R. Taylor, J. Thacker, K. A. Thorne, K. S. Thorne, A. Thuring, K. V. Tokmakov,

C. Torres, C. Torrie, G. Traylor, M. Trias, W. Tyler, D. Ugolini, C. Ungarelli, K. Urbanek, H. Vahlbruch, M. Vallisneri,

C. Van Den Broeck, M. Varvella, S. Vass, A. Vecchio, J. Veitch, P. Veitch, A. Villar, C. Vorvick, S. P. Vyachanin,

S. J. Waldman, L. Wallace, H. Ward, R. Ward, K. Watts, J. Weaver, D. Webber, A. Weber, A. Weidner, M. Weinert, A. Weinstein, R. Weiss, S. Wen, K. Wette, J. T. Whelan, D. M. Whitbeck, S. E. Whitcomb, B. F. Whiting, C. Wilkinson, P. A. Willems, L. Williams, B. Willke, I. Wilmut, W. Winkler, C. C. Wipf, S. Wise, A. G. Wiseman, G. Woan, D. Woods, R. Wooley, J. Worden, W. Wu, I. Yakushin, H. Yamamoto, Z. Yan, S. Yoshida, N. Yunes, M. Zanolin, J. Zhang, L. Zhang, 


\section{P. Zhang, C. Zhao, N. Zotov, M. Zucker, H. zur Muhlen, and J. Zweizig}

(Received 19 July 2007; published 30 July 2007)

DOI: 10.1103/PhysRevD.76.029905

PACS numbers: 04.80.Nn, 04.30.Db, 07.05.Kf, 95.55.Ym, 99.10.Fg

This paper was published online on 9 July 2007 with incorrect affiliation numbering in the author list. The affiliations have been corrected as of 23 July 2007. The text is correct in the printed version of the journal. 